\title{
ARITHMETICITY OF TOTALLY GEODESIC LIE FOLIATIONS WITH LOCALLY SYMMETRIC LEAVES*
}

\author{
RAUL QUIROGA-BARRANCO ${ }^{\dagger}$
}

\begin{abstract}
Zimmer [9] proved that, on a compact manifold, a foliation with a dense leaf, a suitable leafwise Riemannian symmetric metric and a transverse Lie structure has arithmetic holonomy group. In this work we improve such result for totally geodesic foliations by showing that the manifold itself is arithmetic. This also gives a positive answer, for some special cases, to a conjecture of E. Ghys [5].
\end{abstract}

Key words. Semisimple Lie groups, arithmeticity, foliations, transverse structures, tangential structures, pseudoRiemannian geometry.

AMS subject classifications. Primary 53C12, 53C24; Secondary 53C10, 22E46

1. Introduction. In this work we want to consider a compact manifold $M$ with a smooth foliation $\mathcal{F}$. It is well known that for such manifolds, the introduction of transverse structures has played a fundamental role in the theory of foliations. In particular, Lie foliations, i.e. transversely modeled on a Lie group (see Section 2 for more details), are very useful when studying all sorts of transverse geometries. A remarkable case is given by the work of Molino on Riemannian foliations [5]. A natural problem to consider is the classification of Lie foliations on compact manifolds. Nevertheless, this seems to be a problem too general to solve without further considerations.

The following construction provides a fundamental example of a Lie foliation. Let $\pi: L \rightarrow H$ be a surjective homomorphism between connected Lie groups. Then the fibers of $\pi$ yield a foliation on $L$ which is a Lie foliation modeled on $H$. If $\Gamma$ is a cocompact discrete subgroup of $L$, then its left action preserves the Lie foliation on $L$ and so there is an induced Lie foliation on the compact manifold $\Gamma \backslash L$. Moreover, if $K$ is a compact subgroup of the kernel of $\pi$, then the double coset $\Gamma \backslash L / K$ is a compact manifold carrying a Lie foliation modeled in $H$ as well. We will refer to these double coset examples as homogeneous Lie foliations. E. Ghys has conjectured (see Appendix E to [5]) that every Lie foliation can be obtained from a fibration over a homogeneous Lie foliation, as long as the model group $H$ has no compact factors; we refer to page 301 of [5] for the precise statement.

On the other hand, one can study Lie foliations that also carry some sort of leafwise structure. Zimmer [9] did so by considering a compact foliated manifold carrying both a transverse Lie structure and a leafwise Riemannian metric so that the leaves are covered by a symmetric space of noncompact type and higher rank. It was established in [9] the following arithmeticity for the holonomy group of such foliations. We refer to [9] for the complete statement. We observe that the result in [9] is stated for foliations with a simply connected dense leaf, but the existence of one such leaf is guaranteed just from the presence of a dense one by the main result in [8].

ThEOREM 1.1 (Zimmer [9]). Let $H$ be a connected Lie group and $\mathcal{F}$ an $H$ foliation (i.e. with a transverse Lie structure modeled on $H$ ) of a compact manifold

\footnotetext{
${ }^{*}$ Received July 21, 2007; accepted for publication April 18, 2008.

${ }^{\dagger}$ Centro de Investigación en Matemáticas, A. P. 402, Guanajuato, Gto., C.P. 36000, México (quiroga@cimat.mx). Supported by SNI-México, Conacyt Grant 44620 and Concyteg Grant 07-02K662-091.
} 
M. Suppose that $\mathcal{F}$ has a dense leaf. Assume there is a leafwise Riemannian metric on $M$ such that each leaf is isometrically covered by an irreducible symmetric space of noncompact type and rank at least 2 . Then $H$ is semisimple and the holonomy group $\Lambda \subset H$ is a dense arithmetic subgroup, in other words, $\operatorname{Ad}_{H}(\Lambda)$ is commensurable with the image in $\operatorname{Ad}_{H}(H)$ of an arithmetic lattice $\Gamma$ of a semisimple Lie group $L$ with respect to a smooth surjection $L \rightarrow \operatorname{Ad}_{H}(H)$.

Once such notions of arithmeticity had been considered, we can specialize the above construction of a homogeneous Lie foliation as follows. Suppose both $L$ and $H$ are semisimple Lie groups with finite center, and that $\Gamma$ is an arithmetic irreducible lattice. Then the double coset $\Gamma \backslash L / K$ is called an arithmetic manifold, and so we will say that the foliation constructed above from the epimorphism $\pi$ is an arithmetic homogeneous Lie foliation. Note that if $K$ is a maximal compact subgroup of the kernel of $\pi$, say $G$, then $\Gamma \backslash L / K$ carries a leafwise Riemannian metric so that each leaf is isometrically covered by the symmetric space $G / K$.

Given Ghys' conjecture and Zimmer's arithmeticity result, one can consider the problem of determining the relation between Lie foliations with locally symmetric leaves as in Theorem 1.1 and arithmetic homogeneous Lie foliations. The main goal of this work is to prove that any foliation as in Theorem 1.1 which is also totally geodesic is, up to a finite covering, an arithmetic homogeneous Lie foliation. From now on, $G$ denotes a connected noncompact simple Lie group with trivial center and $X_{G}=G / K$ the symmetric space associated to $G$, where $K$ is a maximal compact subgroup of $G$.

MAIN THEOREM . Let $M$ be a compact manifold carrying a smooth foliation $\mathcal{F}$ that admits a transverse Lie structure and a Riemannian metric for which $\mathcal{F}$ is totally geodesic and such that every leaf is isometrically covered by the symmetric space $X_{G}$. Suppose that $\mathcal{F}$ has a dense leaf and $X_{G}$ is irreducible with rank at least 2 . Then, up to a finite covering, $M$ is an arithmetic manifold and $\mathcal{F}$ is an arithmetic homogeneous Lie foliation. More precisely, there exist:

1. a finite covering map $\pi: \widehat{M} \rightarrow M$,

2. a connected semisimple Lie group $H$ with finite center,

3. an arithmetic irreducible lattice $\Gamma$ of $H \times G$, and

4. a diffeomorphism $\varphi: \Gamma \backslash(H \times G) / K=\Gamma \backslash(H \times G / K) \rightarrow \widehat{M}$, where $K$ is a maximal compact subgroup of $G$,

such that $\pi \circ \varphi$ maps the foliation of $\Gamma \backslash(H \times G / K)$ induced by the factor $G / K$ of $H \times G / K$ diffeomorphically onto the foliation of $M$, and so that it preserves both the leafwise Riemannian symmetric metric and the transverse Lie structure.

This result provides a positive answer to Ghys' conjecture in a special case. It also relates to Zimmer's arithmeticity theorem for the holonomy of a Lie foliation since it shows that not just the holonomy but the foliation and the manifold are arithmetic if we consider the case of totally geodesic foliations.

The proof of this result uses our previous work on actions of pseudoRiemannian manifolds (see [7]) and builds on Theorem 1.1. It is already well known that for a manifold $M$ as in the Main Theorem, there is a manifold $M^{*}$ acted upon by $G$ and that fibers, as a principal bundle with compact group, over $M$; this is in fact one of the main objects considered in [9]. Here we show that on such a manifold $M^{*}$ we can build a $G$-invariant pseudoRiemannian metric thus allowing us to apply the results from [7]. As it will be clear from the proof, the compactness in the Main Theorem is only used to ensure completeness of the transverse structures considered. Hence, our 
Main Theorem holds for $M$ noncompact carrying a complete transverse Lie structure and a holonomy invariant transverse smooth measure.

2. Preliminaries on transverse and tangential structures. In this section we assume that $M$ is a manifold equipped with a smooth foliation $\mathcal{F}$. Also, we denote with $T \mathcal{F}$ the tangent bundle to the leaves.

We start by describing some basic facts about transverse structures on $\mathcal{F}$. For these, the fundamental notion is that of the transverse frame bundle $L_{T}(\mathcal{F})$ of the foliation which is defined as the linear frame bundle of $T M / T \mathcal{F}$.

A foliate vector field on $M$ is a smooth vector field $X$ on $M$ such that, for every smooth vector field $Y$ over $M$ tangent to the leaves, the Lie bracket $[X, Y]$ is tangent to the leaves as well. A transverse field for the foliated manifold $M$ is a section $\bar{X}$ of $T M / T \mathcal{F}$ that can be seen as the image under the natural projection $T M \rightarrow T M / T \mathcal{F}$ of a foliate vector field. The vector space of transverse vector fields, denoted with $\mathfrak{l}(M, \mathcal{F})$, is easily seen to inherit a Lie algebra structure from the Lie brackets of foliate vector fields (see [5] for details). We define a transverse parallelism of the foliation $\mathcal{F}$ as a family of pointwise linearly independent transverse fields $\bar{X}_{1}, \ldots, \bar{X}_{q}$, where $q$ is the codimension of $\mathcal{F}$ in $M$. Clearly, a transverse parallelism defines a trivialization of $T M / T \mathcal{F}$ and thus a reduction of $L_{T}(\mathcal{F})$ to the identity group. Conversely, a reduction of $L_{T}(\mathcal{F})$ to the identity group defines a transverse parallelism if the total space of such reduction is saturated for the lifted foliation on $L_{T}(\mathcal{F})$. For the definition of the lifted foliation, the last claim and basic associated notions we refer to [5]. A transverse parallelism is called complete if the transverse vector fields that define it are the image of complete foliate vector fields.

From these remarks, a transverse parallelism defines reductions of $L_{T}(\mathcal{F})$ to both $O(q)$ and $\mathrm{SL}^{ \pm}(q ; \mathbb{R})$ which are saturated with respect to the lifted foliation. Hence, from a transverse parallelism, we obtain a transverse Riemannian structure and a holonomy invariant transverse smooth measure. Note that the former is given by declaring the transverse fields that provide the parallelism as pointwise orthonormal. Furthermore, the transverse measure defined by the transverse Riemannian metric and the transverse measure given by the $\mathrm{SL}^{ \pm}(q ; \mathbb{R})$-reduction of $L_{T}(\mathcal{F})$ are easily seen to be the same.

From now on, for a foliation $\mathcal{F}$, a transverse Lie structure modeled on a connected Lie group $H$ (or an $H$-foliation) will be given by an open cover $\left\{U_{i}\right\}_{i}$ of $M$ and smooth submersions $f_{i}: U_{i} \rightarrow H$, compatible with the foliation, onto open subsets of $H$ so that for every $i, j$ there is some $x_{i j} \in H$ for which we have:

$$
f_{i}=L_{x_{i j}} \circ f_{j}
$$

on $U_{i} \cap U_{j}$, where $L_{x}$ is the left translation in $H$ by a given $x \in H$.

Alternatively, an $H$-foliation on $\mathcal{F}$ can be defined as a transverse parallelism $\bar{X}_{1}, \ldots, \bar{X}_{q}$ whose linear span over $\mathbb{R}$ is a Lie algebra isomorphic to the Lie algebra $\mathfrak{h}$ of $H$ (see [5]). Given this remark, we say that a transverse Lie structure is complete if the completeness condition holds for the corresponding transverse parallelism. Also, observe that the transverse Riemannian structure given by the parallelism of a transverse Lie structure modeled on the group $H$ corresponds to a choice of a left invariant metric on $H$.

Finally, we refer to $[5,9]$ and their references for a more precise account of the basic notions associated to transverse Lie structures, including those of a development, holonomy representation and holonomy group of a Lie foliation. We will use freely 
the basic facts of the theory of transverse Lie structures, providing references when necessary.

For the notion of tangential structure for totally geodesic foliations, dual to the notion of transverse structure, the fundamental object is $L_{t g}(\mathcal{F})$, the linear frame bundle of the vector bundle $T \mathcal{F}$. For the rest of this section we assume that $M$ carries a Riemannian metric for which $\mathcal{F}$ is totally geodesic but with otherwise arbitrary geometry along the leaves.

One of the most useful properties of totally geodesic foliations is the fact that the leaves are isometrically unchanged as we move perpendicularly to them. More precisely, if $\gamma:[0,1] \rightarrow M$ is a curve starting at $x$ and everywhere perpendicular to $\mathcal{F}$, then there exists a unique family of isometries $\left(\psi_{t}\right)_{t \in[0,1]}$ such that $\psi_{t}: V_{x} \rightarrow V_{\gamma(t)}$, where $V_{\gamma(t)}$ is a neighborhood of $\gamma(t)$ in the leaf that contains $\gamma(t), \psi_{0}$ is the identity map and the curves $y \mapsto \psi_{t}(y)$ are everywhere perpendicular to $\mathcal{F}$ for every $y \in V_{x}$ (see [3] for details). The isometries $\left(\psi_{t}\right)_{t}$ are called the elements of horizontal holonomy associated to $\gamma$.

Denote with $T \mathcal{F}^{\perp}$ the orthogonal complement of $T \mathcal{F}$. Then, the vector bundle $T \mathcal{F}^{\perp}$ is lifted to a vector subbundle $\mathcal{H}$ of $T L_{t g}(\mathcal{F})$, called the lifted horizontal bundle, as follows (see $[1,2]$ for further details). For a given $v \in T_{x} \mathcal{F}^{\perp}$ choose a curve $\gamma:[0,1] \rightarrow M$ starting at $x$ with velocity $v$ and everywhere perpendicular to $\mathcal{F}$. Let $\left(\psi_{t}\right)_{t}$ be the elements of horizontal holonomy associated to $\gamma$. If $\alpha \in L_{t g}(\mathcal{F})$ is an element in the fiber over $x$, then the curve $\bar{\gamma}(t)=d\left(\psi_{t}\right)_{x} \circ \alpha$ lies in $L_{t g}(\mathcal{F})$ and its velocity vector $\bar{v}=\bar{\gamma}^{\prime}(0)$ is called the horizontal lift of $v$ at $\alpha$. The set of all such horizontal lifts of elements of $T_{x} \mathcal{F}^{\perp}$ to the point $\alpha$ is by definition the fiber $\mathcal{H}_{\alpha}$ of the bundle $\mathcal{H}$. The lifted horizontal bundle is used to define tangential structures. More precisely, for a subgroup $F$ of $\mathrm{GL}(p)$ (where $p$ is the dimension of the leaves of $\mathcal{F}$ ), a tangential $F$-structure for $(M, \mathcal{F})$ is an $F$-reduction $Q$ of $L_{t g}(\mathcal{F})$ such that $\mathcal{H}_{\alpha} \subset T_{\alpha} Q$ for every $\alpha \in Q$. For $F=\{e\}$, the tangential structure is called a tangential parallelism. Another example is given by $O_{t g}(\mathcal{F})$, the principal fiber bundle of orthonormal frames for $T \mathcal{F}$, which defines a tangential $O(p)$-structure. On the other hand, the pull-back in $O_{t g}(\mathcal{F})$ of the leaves of $\mathcal{F}$ defines a foliation $\mathcal{F}_{t g}$. The bundle $T \mathcal{F}_{t g}$ can be endowed with a natural parallelism given by the standard horizontal vector fields with respect to the Levi-Civita connection along the leaves and the vector fields defined by the $O(p)$-action on $O_{t g}(\mathcal{F})$.

3. A $G$-space associated to $M$. In this section we recall the construction of a manifold fibered over $M$ by a compact group and on which there is an action of the group $G$, for $M$ and $G$ as in the Main Theorem. Our main contribution here is the construction of a pseudoRiemannian metric which turns out to be $G$-invariant. The next result is essentially Proposition 2.5 from [9] with an additional final claim which we explain.

Proposition 3.1. Let $M$ be a manifold with a smooth foliation and a leafwise Riemannian metric such that every leaf is isometrically covered by $X_{G}$. Then there exists a principal right $K$-bundle $\pi_{0}: M^{*} \rightarrow M$, where $K$ is a maximal compact subgroup of $G$, so that the $K$-action on $M^{*}$ extends to a locally free right $G$-action on $M^{*}$, which thus defines a foliation whose leaves are precisely the inverse images under $\pi_{0}$ of the leaves of $M$. Furthermore, if $M$ has a dense leaf, then $M^{*}$ has a dense G-orbit. Finally, any transverse geometric structure defined on $M$ induces a corresponding $G$-invariant transverse structure on $M^{*}$ which is preserved by $\pi_{0}$. 
Proof. From [9] only the last claim requires some justification. For that we recall that transverse structures can be defined by submersions into suitable geometric spaces, as in our description of Lie structures above. Then, it is enough to compose with $\pi_{0}$ the local submersions that define a transverse structure on $M$. The $G$ invariance follows easily from the first part of the proposition.

From now on and unless otherwise stated, $M$ will denote a manifold satisfying the hypotheses of the Main Theorem. Also, $M^{*}$ will denote the manifold obtained applying Proposition 3.1 to $M$. In particular, $M^{*}$ carries a $G$-invariant transverse Lie structure modeled in the same group as that for $M$.

We now describe a $G$-invariant pseudoRiemannian metric on $M^{*}$ that plays a fundamental role to prove our results.

There is a leafwise pseudoRiemannian metric on $M^{*}$ so that each leaf is isometrically covered by $G$ with the bi-invariant pseudoRiemannian metric induced from the Killing form of its Lie algebra. The latter is easily seen by observing that every leaf of the foliation on $M^{*}$ is $G$-equivariantly diffeomorphic to a quotient $F \backslash G$, for some discrete subgroup $F$.

For $M$ satisfying the hypotheses of the Main Theorem, Proposition 3.1 shows that $M^{*}$ carries a $G$-invariant transverse Lie structure. As we observed in Section 2, this induces a transverse Riemannian structure by choosing a left invariant Riemannian metric on the simply connected Lie group that models the transverse Lie structure. In what follows, we fix one such transverse Riemannian structure.

Our next goal is to show that such leafwise pseudoRiemannian structure and transverse Riemannian structure can be tied together into a $G$-invariant pseudoRiemannian metric defined on $M^{*}$. The main tools will come from the fact that $\mathcal{F}$ is totally geodesic. We start by proving that $M^{*}$ defines a tangential structure for $M$. From now on, we fix a maximal compact subgroup $K$ of $G$ and denote $x_{0}=e K \in G / K=X_{G}$.

Proposition 3.2. If $O_{t g}(\mathcal{F})$ is considered as the bundle of linear isometries from $T_{x_{0}} X_{G}$ onto the fibers of $T \mathcal{F}$, then the map $M^{*} \rightarrow O_{t g}(\mathcal{F})$ given by $\varphi \mapsto d \varphi_{x_{0}}$ realizes $M^{*}$ as a tangential $K$-structure of $\mathcal{F}$.

Proof. Since every isometry is completely determined by its 1-jet at any given point, the given map is an embedding. Also, if we consider the homomorphism from $K$ into $O\left(T_{x_{0}} X_{G}\right) \simeq O(p)\left(p=\operatorname{dim} X_{G}\right)$ given by $\varphi \mapsto d \varphi_{x_{0}}$, such embedding is clearly equivariant and so $M^{*}$ is a $K$-reduction of $O_{t g}(\mathcal{F})$. It remains to show that $\mathcal{H}_{\alpha} \subset T_{\alpha} M^{*}$ for every $\alpha \in M^{*}$.

Let $\alpha \in M^{*}$ be given and suppose that $\alpha$ projects to $x$ in $M$, so that $\alpha: T_{x_{0}} X_{G} \rightarrow$ $T_{x} \mathcal{F}$. For a given curve $\gamma:[0,1] \rightarrow M$ starting at $x$ and perpendicular to $\mathcal{F}$, let $\left(\psi_{t}\right)_{t}$ be the elements of horizontal holonomy associated to $\gamma$. Then for the curve $\bar{\gamma}(t)=d\left(\psi_{t}\right)_{x} \circ \alpha$ we have $\bar{\gamma}^{\prime}(0) \in \mathcal{H}_{\alpha}$ and every element in $\mathcal{H}_{\alpha}$ is of this form; this is a consequence of the fact that $\mathcal{F}$ is totally geodesic (see [1]). Hence, it suffices to show that $\bar{\gamma}$ lies in $M^{*}$ for any such $\alpha$ and $\gamma$. Let $\varphi \in M^{*}$ be such that $\varphi\left(x_{0}\right)=x$ and $d \varphi_{x_{0}}=\alpha$. In particular, we have $\bar{\gamma}(t)=d\left(\psi_{t} \circ \varphi\right)_{x_{0}}$ for every $t$. We recall that a small enough neighborhood of a locally symmetric space can be isometrically embedded in a (global) symmetric space. Hence, if the neighborhoods $V_{\gamma(t)}$ are small enough, for every $t$ there exists an isometric covering $\varphi_{t}: X_{G} \rightarrow L_{\gamma(t)}$ onto the leaf $L_{\gamma(t)}$ containing $\gamma(t)$ such that $\varphi_{t}=\psi_{t} \circ \varphi$ in a neighborhood of $x_{0}$ in $X_{G}$. In particular, $\bar{\gamma}(t)=d\left(\varphi_{t}\right)_{x_{0}}$ and since $\varphi_{t} \in M^{*}$ for every $t$, we conclude that $\bar{\gamma}$ lies in $M^{*}$ as required. 
From the previous result it follows that the lifted horizontal bundle $\mathcal{H}$ restricted to $M^{*}$ is tangent to $M^{*}$. We will denote such restriction with $\mathcal{H}^{*}$. In order to further understand the properties of $\mathcal{H}^{*}$ we will need the following elementary fact about symmetric spaces. For the definition of standard horizontal vector fields on the frame bundle of a Riemannian manifold we refer to [4].

LemMa 3.3. Let $O\left(X_{G}\right)$ be the orthonormal frame bundle of $X_{G}$ viewed as the space of linear isometries of $T_{x_{0}} X_{G}$ onto the fibers of $T X_{G}$. Then, for the embedding $G \hookrightarrow O\left(X_{G}\right)$ given by $g \mapsto d g_{x_{0}}$, the standard horizontal vector fields on $O\left(X_{G}\right)$ are tangent to $G$. Also, if $X=\Gamma \backslash X_{G}$ is a Riemannian quotient of $X_{G}$, where $\Gamma \subset G$ is discrete, then there is an induced embedding $\Gamma \backslash G \hookrightarrow O(X)$ so that the standard horizontal vector fields are tangent to $\Gamma \backslash G$.

Proof. First we observe that the embedding $G \hookrightarrow O\left(X_{G}\right)$ is $G$-equivariant for the natural left $G$-action on $G$ and the $G$-action on $O\left(X_{G}\right)$ that lifts from that on $X_{G}$.

Choose $v \in T_{x_{0}} X_{G}$ and denote with $\left(T_{t}\right)_{t}$ the one-parameter subgroup of transvections of $X_{G}$ along the geodesic $\gamma_{v}$ with initial velocity vector $v$; we recall that a transvection along a geodesic is an isometry that leaves invariant the geodesic and whose derivative defines the parallel transport along such geodesic. Thus $\widehat{\gamma}_{v}(t)=d\left(T_{t}\right)_{x_{0}}$ yields the parallel transport along the geodesic $\gamma_{v}$ and so it is the horizontal lift at $\alpha_{0}$ in $O\left(X_{G}\right)$ with respect to the Levi-Civita connection, where $\alpha_{0} \in O\left(X_{G}\right)$ is the identity map of $T_{x_{0}} X_{G}$. Hence, $\widehat{v}=\widehat{\gamma}_{v}^{\prime}(0)$ is horizontal at $\alpha_{0}$; moreover all horizontal vectors at $\alpha_{0}$ are obtained through this construction. Since $T_{t} \in G$ for every $t$, then $\widehat{\gamma}_{v}$ lies in $G$ with respect to the given embedding. Hence, $T_{e} G$ contains the horizontal subspace of $O\left(X_{G}\right)$ at $\alpha_{0}$ defined by the Levi-Civita connection. Since the embedding $G \hookrightarrow O\left(X_{G}\right)$ is $G$-equivariant and the $G$-action preserves the Levi-Civita connection on $O\left(X_{G}\right)$ the first part follows. The last claim follows by modding out by the left $\Gamma$-action and using the properties obtained so far.

Proposition 3.4. The lifted horizontal bundle $\mathcal{H}^{*}$ on $M^{*}$ is $G$-invariant.

Proof. Let $\left(X_{i}\right)_{i=1}^{p}$ be the basis for the standard horizontal vector fields with respect to the Levi-Civita connection along the leaves of $\mathcal{F}$ and corresponding to the canonical basis of $\mathbb{R}^{p}$. Let $\left(A_{i}\right)_{i=1}^{l}$ be the vertical fields of the bundle $M^{*} \rightarrow M$ that come from the right action of the one-parameter subgroups defined by a basis of $\mathfrak{k}$ (the Lie algebra of $K)$. For such choices, denote $\mathcal{P}=\left(X_{1}, \ldots, X_{p}, A_{1}, \ldots, A_{l}\right)$; then, $\mathcal{P}$ consists of restrictions to $M^{*}$ of a subset of the natural tangential parallelism of $O_{t g}(\mathcal{F})$ (see $\left.[2]\right)$. Also, by Proposition 3.2 , the set $\mathcal{P}$ defines a parallelism for the foliation by $G$-orbits in $M^{*}$.

Also, from the proof of Lemma 3.3, the last claim in Proposition 3.1 and the fact that $G$ preserves the horizontal standard vector fields on $X_{G}$, it follows that the elements in $\mathcal{P}$ are locally given by left invariant vector fields on $G$. We recall the (elementary) fact that on a Lie group the left invariant vector fields have flows corresponding to right actions of one-parameter subgroups. From this fact, again the last claim of Proposition 3.1 and since $\mathcal{P}$ is a parallelism, it is easy to see that $\mathcal{P}$ consists of vector fields whose local flows generate the right $G$-action on $M^{*}$.

Let $v^{*} \in \mathcal{H}^{*}$ be given. Choose curves $\gamma$ in $M$ (perpendicular to $\mathcal{F}$ ) and $\bar{\gamma}$ in $M^{*}$ as in the proof of Proposition 3.2, so that $\bar{\gamma}^{\prime}(0)=v^{*}$ and $\bar{\gamma}(t)=d\left(\psi_{t}\right)_{x} \circ \alpha$, where $\left(\psi_{t}\right)_{t}$ are the elements of horizontal holonomy associated to $\gamma, \alpha$ is the basepoint of $v^{*}$ and $x$ is the projection of $\alpha$. Clearly, the curves $\delta_{y}(t)=\psi_{t}(y)$, where $y \in V_{x}$ with our previous notation, are the integral curves of a local vector field $Z^{*}$ such that $Z_{\alpha}^{*}=v^{*}$; furthermore, $Z^{*}$ is a section of $\mathcal{H}^{*}$ (see [1]). Since the local flow of $Z^{*}$ is given by 
maps of the form $y \mapsto \delta_{y}(t)$ and the latter restrict to the maps $\left(\psi_{t}\right)_{t}$ along the leaves of $\mathcal{F}$, it follows that the flow of $Z^{*}$ fixes the elements in $\mathcal{P}$. Hence, every element of $\mathcal{P}$ commutes with $Z^{*}$, and so the flows given by the elements in $\mathcal{P}$ fix $Z^{*}$. Since such flows generate the $G$-action, then $G$ maps $v^{*}$ into $\mathcal{H}^{*}$ from which the result follows.

As remarked in the proof of Proposition 3.4, the elements in $\mathcal{P}$ are locally given by left invariant vector fields. Hence, by recalling the properties of the metric in $X_{G}$ in terms of $G$, we can consider a pseudoRiemannian metric $h_{1}$ along the leaves of $\mathcal{F}_{t g}$ in $M^{*}$ such that the linear span of the elements in $\mathcal{P}$ are isometric to $\mathfrak{g}$ (the Lie algebra of $G$ ) with its Killing form. Since the latter is invariant under the adjoint action of $G$, it is easy to prove that $h_{1}$ along the foliation $\mathcal{F}_{t g}$ is $G$-invariant. On the other hand, from the $G$-invariance of $\mathcal{H}^{*}$ given by Proposition 3.4, and since $M$ carries a $G$-invariant transverse Riemannian structure, there exists a Riemannian metric $h_{2}$ on $\mathcal{H}^{*}$ which is $G$-invariant. Note that the tangent bundle to the $G$-orbits on $M^{*}$ is $T \mathcal{F}_{t g}$. Since $G$ preserves the decomposition $T M^{*}=T \mathcal{F}_{t g} \oplus \mathcal{H}^{*}$ it follows that $\left(T \mathcal{F}_{t g}, h_{1}\right) \oplus\left(\mathcal{H}^{*}, h_{2}\right)$ defines a $G$-invariant pseudoRiemannian metric $h$ on $M^{*}$. From these remarks we obtain the following result.

Proposition 3.5. Let $M$ satisfy the hypotheses of the Main Theorem. Then, the manifold $M^{*}$ carries a $G$-invariant pseudoRiemannian metric $h$ that satisfies the following properties:

1. Every $G$-orbit is nondegenerate and the metric induced on the G-orbits corresponds to the bi-invariant metric on $G$ given by the Killing form of its Lie algebra.

2. The metric $h$ defines transverse Riemannian structure that corresponds to the one given by the transverse Lie structure as described in Section 2.

In what follows, $h$ will denote the pseudoRiemannian metric on $M^{*}$ described by the previous proposition.

4. Proof of the Main Theorem. One of our main tools is the following result from [7].

THEOREM $4.1([7])$. Let $G$ be a connected noncompact simple Lie group. If $G$ acts faithfully and topologically transitively on a compact manifold $X$ preserving a pseudoRiemannian metric such that $n_{0}=m_{0}$, then the $G$-action on $X$ is ergodic and engaging, and there exist:

(1) a finite covering $\widehat{X} \rightarrow X$,

(2) a connected Lie group $L$ that contains $G$ as a factor,

(3) a cocompact discrete subgroup $\Gamma$ of $L$ and a compact subgroup $K_{1}$ of $C_{L}(G)$, for which the G-action on $X$ lifts to $\widehat{X}$ so that $\widehat{X}$ is G-equivariantly diffeomorphic to $K_{1} \backslash L / \Gamma$. Furthermore, there is an ergodic and engaging $G$-invariant finite smooth measure on $L / \Gamma$.

Let $h$ be the $G$-invariant pseudoRiemannian metric on $M^{*}$ constructed in Section 3. Following [7] we define $n_{0}$ and $m_{0}$ as the maximal dimensions of the null tangent subspaces for $G$ and $M^{*}$, respectively; for $G$ we consider the bi-invariant pseudoRiemannian metric given by the Killing form of its Lie algebra. Since the metric $h$ restricted to the $G$-orbits is precisely this bi-invariant metric and $h$ is Riemannian in the orthogonal complement to the tangent bundle to the $G$-orbits, it follows easily that $n_{0}=m_{0}$. On the other hand, by Proposition 3.1 there is a dense $G$-orbit in $M^{*}$. Hence the hypotheses of Theorem 4.1 are satisfied. It follows that there exists a 
finite covering $\pi^{*}: \widehat{M^{*}} \rightarrow M^{*}$ over which the $G$-action can be lifted so that there is a $G$-equivariant diffeomorphism:

$$
\varphi^{*}: \Gamma \backslash(N \times G) \rightarrow \widehat{M^{*}},
$$

where $N$ is a homogeneous Riemannian manifold and $\Gamma \subset \operatorname{Iso}(N) \times G$. That $N$ is Riemannian follows from the fact that the orthogonal complement to the $G$-orbits is Riemannian in our construction of the metric $h$ on $M^{*}$. Note that without loss of generality we can assume that $N$ is simply connected by replacing the covering $N \times G \rightarrow \widehat{M^{*}}$ with its composition with the universal covering map of $N$ in the first factor.

From this discussion, the manifolds $N \times \widetilde{G}$ and $\widetilde{M^{*}}$ are $\widetilde{G}$-equivariantly diffeomorphic. Let $H$ be a Lie group that models the transverse Lie structure of $\widehat{M^{*}}$. By Theorem 1.1 the group $H$ is semisimple. Choose a development $D: N \times \widetilde{G} \cong \widetilde{M^{*}} \rightarrow H$. In other words, $D$ is a submersion equivariant with respect to the holonomy representation $\rho: \pi_{1}\left(\widehat{M^{*}}\right) \rightarrow H$. Clearly, we can assume that $H$ is simply connected.

The foliation in $\widehat{M^{*}}$ is given by the $G$-orbits, and so the foliation in $N \times \widetilde{G \cong \widetilde{M^{*}}}$ is given by the $\widetilde{G}$-orbits. Since $D$ is a development, it follows that the connected components of the fibers of $D$ are the $\widetilde{G}$-orbits. Hence, the restriction $\left.D\right|_{N \times\{e\}}$ defines a local diffeomorphism $N \rightarrow H$. Moreover, by the choice of $N$ and its Riemannian metric as given in Proposition 3.5, such map is a local isometry, hence (by Corollary 29 in page 202 in [6]) a covering map and so a diffeomorphism. It follows that $N$ admits a Lie group structure so that the map $\left.D\right|_{N \times\{e\}}: N \rightarrow H$ is an isomorphism. Hence, if we consider $N$ endowed with such Lie group structure, then the canonical projection $N \times \widetilde{G} \rightarrow N$ is a development for the transverse Lie structure of $\widehat{M^{*}}$. In particular, we can replace the Riemannian manifold $N$ with the Lie group $H$ carrying a suitable left invariant Riemannian structure.

At the same time, since the deck transformation group of the covering $\widetilde{G} \rightarrow G$ is a central subgroup of $\widetilde{G}$ and $\pi_{1}\left(\widehat{M^{*}}\right) \subset \operatorname{Iso}(H) \times \widetilde{G}$, then using the diffeomorphism $\varphi^{*}$ above it follows that the natural projection $H \times G \rightarrow H$ defines a development for the transverse Lie structure on $\widehat{M^{*}}$ as well.

On the other hand, if $\gamma \in \Gamma$, then we can write $\gamma=\left(\gamma_{1}, \gamma_{2}\right)$, where $\gamma_{1}$ is a diffeomorphism of $H$ and $\gamma_{2} \in G$. Since $\pi$ is equivariant with respect to the corresponding holonomy representation $\rho: \Gamma \rightarrow H$, we conclude that:

$$
\gamma_{1}(x)=D\left(\gamma_{1} x, \gamma_{2} g\right)=D(\gamma(x, g))=\rho(\gamma) D(x, g)=y x
$$

is satisfied for some $y \in H$, depending only on $\gamma$, and every $(x, g) \in H \times G$. It follows that $\gamma_{1}$ is the left translation by an element in $H$ and with respect to this we have $\gamma_{1} \in H$, thus showing that $\Gamma$ is a subgroup of $H \times G$.

Next, if we consider the finite covering:

$$
\pi^{*}: \Gamma \backslash(H \times G) \rightarrow M^{*}
$$

just constructed, then for $K$ a maximal compact subgroup of $G$ as before, we observe that taking the quotient on this map by the $K$-action on the right, we obtain a finite covering map:

$$
\pi: \Gamma \backslash(H \times G / K) \rightarrow M,
$$

where $G / K$ is the symmetric space that isometrically covers the leaves of the foliation in $M$. Hence, from the previous discussion most of the claims of the Main Theorem 
have now been proved. It remains to show that we can choose our covering $\pi$ so that $H$ is replaced by a finite center quotient group; this is achieved by a modding out by a suitable central subgroup of $H$ through a standard procedure which already appears in Section 6 of [7]. Finally, the arithmeticity of $\Gamma$ is a consequence of Margulis' arithmeticity theorem.

\section{REFERENCES}

[1] R. A. Blumenthal and J. J. HeBda, Complementary distributions which preserve the leaf geometry and applications to totally geodesic foliations, Quart. J. Math. Oxford Ser. (2), 35 (1984), pp. 383-392.

[2] G. CaIrns, Géométrie globale des feuilletages totalement géodésiques, C. R. Acad. Sci. Paris Sér. I Math., 297 (1983), pp. 525-527.

[3] D. L. Johnson And L. B. WhitT, Totally geodesic foliations, J. Differential Geom., 15 (1980), pp. 225-235.

[4] S. Kobayashi and K. Nomizu, Foundations of differential geometry, vol. I. Reprint of the 1969 original. Wiley Classics Library. A Wiley-Interscience Publication. John Wiley \& Sons, Inc., New York, 1996.

[5] P. Molino, Riemannian foliations. Translated from the French by Grant Cairns. With appendices by Cairns, Y. Carriere, É. Ghys, E. Salem and V. Sergiescu. Progress in Mathematics, 73. Birkhauser Boston, Inc., Boston, MA, 1988.

[6] B. O'NeILl, Semi-Riemannian geometry: With applications to relativity, Pure and Applied Mathematics, 103, Academic Press, Inc. [Harcourt Brace Jovanovich, Publishers], New York-London, 1983.

[7] R. Quiroga-BARranco, Isometric actions of simple Lie groups on pseudoRiemannian manifolds, Ann. of Math. (2), 164 (2006), pp. 941-969.

[8] G. Stuck, R. J. Zimmer, Stabilizers for ergodic actions of higher rank semisimple groups, Ann. of Math. (2), 139 (1994), pp. 723-747.

[9] R. J. Zimmer, Arithmeticity of holonomy groups of Lie foliations, J. Amer. Math. Soc., 1 (1988), pp. 35-58. 
R. QUIROGA-BARRANCO 